\title{
The modulation relationship of genomic pattern of intratumor heterogeneity and immunity microenvironment heterogeneity in hepatocellular carcinoma
}

\author{
LIU-BO LI ${ }^{1}$, LU YANG ${ }^{2}$, GUO-QUN XIE ${ }^{1}$, XIAO-CUI ZHOU ${ }^{1}$, XU-BO SHEN ${ }^{1}$, \\ QIU-LIN XU ${ }^{1}$, ZHENG-YUAN MA ${ }^{2}$ and XIAO-DONG GUO ${ }^{1}$ \\ ${ }^{1}$ Department of Oncology, Yueyang Hospital of Integrated Traditional Chinese and Western Medicine \\ Affiliated to Shanghai University of Traditional Chinese Medicine, Shanghai 200080; \\ ${ }^{2}$ Shanghai Information Center for Life Sciences, Shanghai Institute of Nutrition and Health, \\ University of Chinese Academy of Sciences, Chinese Academy of Sciences, Shanghai 200031, P.R. China
}

Received January 16, 2020; Accepted July 15, 2020

DOI: $10.3892 / \mathrm{ol} .2020 .12096$

\begin{abstract}
Hepatocellular carcinoma (HCC) is the fifth most common cancer in the world, with the second highest mortality rate among all cancer types. Growing evidence has demonstrated the notable effects of intratumor heterogeneity (ITH) and tumor immune microenvironment heterogeneity (TIMH) on the biological processes involved in HCC. However, the interactive mechanisms between ITH and TIMH is still unclear. The present study systematically screened the mRNA expression, simple nucleotide variation data and clinical data of samples from The Cancer Genome Atlas (TCGA). The mutant-allele tumor heterogeneity (MATH) score was used to represent ITH, and TCGA cohort was divided into two groups according to the MATH score. Next, different immune-related signaling pathways and enriched immune-related genes were identified using Gene Set Enrichment Analysis of these two groups, and the results revealed that interleukin-1 $\alpha$ (IL1A) and serine/threonine-protein kinase PAK4 were associated with prognosis. Furthermore, CIBERSORT was utilized to calculate the fractions of 22 types of leukocytes to represent TIMH, and the fractions of M1 and M2 macrophages were
\end{abstract}

Correspondence to: Professor Xiao-Dong Guo, Department of Oncology, Yueyang Hospital of Integrated Traditional Chinese and Western Medicine Affiliated to Shanghai University of Traditional Chinese Medicine, 110 Ganhe Road, Shanghai 200080, P.R. China E-mail: gxd1996@shutcm.edu.cn

Professor Zheng-Yuan Ma, Shanghai Information Center for Life Sciences, Shanghai Institute of Nutrition and Health, University of Chinese Academy of Sciences, Chinese Academy of Sciences, 319 Yueyang Road, Shanghai 200031, P.R. China

E-mail: zyma@sibs.ac.cn

Key words: immunity microenvironment, intratumor heterogeneity, hepatocellular carcinoma, tumor-infiltrating lymphocytes, The Cancer Genome Atlas confirmed to be associated with prognosis. Therefore, PAK4, interleukin-1 $\alpha$ (IL1A), and M1/M2 ratio were selected as the key factors involved in the interaction between ITH and TIMH. Afterwards, microRNAs (miRNAs) that were linearly related to the M1/M2 ratio and the potential target genes of the miRNAs were screened. Finally, the regulatory network between PAK4, IL1A, and the M1/M2 ratio was established, bridged by the above miRNAs and the target genes. In addition, PAK4, heat shock protein $105 \mathrm{kDa}$ and miRNA-1911 were demonstrated to be a key factor involved in immune response via Weighted Correlation Network Analysis in HCC.

\section{Introduction}

Hepatocellular carcinoma (HCC) is the fifth most common cancer in the world, with the second highest mortality rate among cancers (1). In HCC treatment, systemic treatment is mostly indispensable, and molecular targeted therapy is an important part (2). Targeted drugs, such as sorafenib, can effectively prolong the survival of patients for several months; however, occasional treatment resistance can still bring about tumor recurrence or progression (3). In addition, immunotherapy has played a key role in the treatment of tumors, such as melanoma and non-small-cell lung cancer, and has achieved initial success in the treatment of $\operatorname{HCC}(4,5)$. Increasing evidence indicates that treatment resistance and tumor response to anti-programmed cell death protein are highly dependent on tumor heterogeneity, immunogenicity and the lymphocyte infiltration in immune microenvironment (6-8).

Intratumor heterogeneity (ITH), the result of tumor cell mutation, is closely associated with neoantigen generation and tumor immunogenicity (9). The interaction between tumor cells and the immune microenvironment is also a reason for ITH (10). Since ITH represents the cell division and proliferation of tumor cells, it is also considered a biological process in tumor progression and is one of the characteristics of malignant tumors (11). Clinical studies have also shown that ITH has been linked to cancer progression and affects the tumor immune response and tumor immune escape $(12,13)$. 
Mutant-allele tumor heterogeneity (MATH) is an algorithm for quantifying ITH that was deduced from whole-exome sequencing data (7). Mroz and Rocco (14) first applied the MATH algorithm to quantify ITH and reported that the prognosis of neck squamous cell carcinoma is related to the MATH score. Subsequent studies have calculated the MATH score in other tumors, and the results verified the clinical outcome and demonstrated that the MATH score is a useful biomarker for the metastasis of cancer, such as colon cancer (15). In a study of breast cancer, Ma et al (6) reported that a high MATH score corresponds with a worse prognosis. Wang et al (16) evaluated ITH in diffuse large B-cell lymphoma using MATH and demonstrated that a higher MATH score indicated an increased risk of progression. Therefore, the MATH score of ITH is a potentially quantitative indicator for various tumors.

Moreover, the occurrence and development of HCC is also closely related to tumor immune microenvironment heterogeneity (TIMH), which is created by the interaction of immune cells, fibroblasts, endothelial cells, mesenchymal stem cells and pericytes with tumor cells (17). In TIMH, cytokines, chemokines and pro-angiogenic and anti-inflammatory mediators produced in lymphocytes or tumor cells play a key role in the tumor immune response $(18,19)$. On the other hand, the recruitment and localization of lymphocytes in the tumor stroma affects the production of cytokines and immune factors, which can be used to predict prognosis (20). TIMH is associated with the type and proportion of lymphocytes in the tumor microenvironment (21). The CIBERSORT method (https://cibersort. stanford.edu/) is an algorithm used to evaluate gene expression data from tumor RNA sequences and analyze the distribution of various types of immune cells inside the sample (22). Based on previous studies $(23,24)$, the CIBERSORT method was used to research TIMH in the present study.

To date, the mechanism between ITH and TIMH remains unclear, and to the best of our knowledge there have been no reports as to whether the MATH score can be used as a biomarker in HCC research. Therefore, the present study used the MATH score to represent ITH and utilized CIBERSORT to calculate the fractions of 22 types of leukocytes in tumor tissues to analyze the intrinsic relationship between ITH and TIMH.

\section{Materials and methods}

Data acquisition and calculation of MATH score. The TCGA-LIHC (Liver hepatocellular carcinoma) multi-data were downloaded from The Cancer Genome Atlas (TCGA) website (https://portal.gdc.cancer.gov/) (accessed on June 20, 2019). From these data, 424 files of RNA-sequencing (RNA-seq) were obtained, of which 50 were paracancerous samples and 374 were HCC samples. Next, the gene expression matrix was extracted through R version 3.5.1 (https://www.r-project.org/). Then, gene symbols were acquired by matching the Ensembl ID from the Ensembl dataset (http://asia.ensembl.org/index.html). The simple nucleotide variation (SNV) data of the 374 patients were obtained from TCGA as Mutation Annotation Forma (MAF) files, and the clinical data were acquired as BCR XML files. Here, 'maftools' packages of R were applied to analyze the MAF files and to calculate the MATH score. In this MATH algorithm, a clustering analysis algorithm was added, and some outliers on the variant allele frequency were removed based on the conventional algorithm to increase the accuracy of the algorithm. Finally, the RNA expression matrix, clinical data and all cohort MATH scores were acquired.

Exploring oncological features and tumor immunology. Based on the MATH score, cohort samples were divided into low and high MATH score groups and the cut-off value was set as 19.39. Kaplan-Meier survival analysis was used to explore the relationship between MATH score and clinical prognosis, and the optimal cut-off value (set as 19.39) was calculated by the 'survminer' package.

To explore the oncological features and gene sets enriched in these two groups, Gene Set Enrichment Analysis (GSEA) was performed to resolve the underlying molecular mechanisms and Kyoto Encyclopedia of Genes and Genomes (KEGG) pathways of enriched gene sets. GSEA was used to investigate the potential mechanisms involving the Molecular Signatures Database gene sets c2 (c2.cp.kegg.v6.1.symbols and c2.cp.biocarta.v6.1.symbols) and c5 (c5.bp.v6.1.symbols) using the JAVA program (http://software.broad institute. org/gsea/index.jsp) (25). One thousand random sample permutations were performed, and the significance threshold was set at $\mathrm{P}<0.05$. After GSEA, the immune-related signaling pathways were selected from the differential signaling pathways. Next, immune-related genes were extracted from those immune-related pathways to explore tumor immunology.

In addition, some immunosuppression-related genes (indoleamine 2,3-dioxygenase interleukin-10, programmed cell death 1 ligand 1 and transforming growth factor- $\beta$ ) were subjected to Kaplan-Meier survival analysis and Pearson's correlation analysis in the group with more immune-related pathways to observe whether those genes affect prognosis.

Selection of immune- and survival-related (IS) genes. First, IS genes were selected using Kaplan-Meier and Cox multivariate regression analysis using the 'survival' and 'survimer' packages of $\mathrm{R}$ software, in which the significance threshold was set at $\mathrm{P}<0.05$.

Second, coexpression analysis, cluster analysis and Pearson's correlation coefficient analysis of immune checkpoints and IS genes were implemented to analyze the positive and negative regulation of these genes in tumor-associated immunity based on ITH.

Analysis of TIMH. In this part, Genotype-Tissue Expression (GTEx) data was downloaded to integrate analysis with TCGA data via UCSC dataset (http://hgdownload.soe.ucsc.edu/downloads.html). Gene expression arrays were mined through CIBERSORT to estimate the fractions of 22 types of leukocyte infiltrates in all samples, and the algorithm was run with 800 permutations. By analyzing the difference in the percentage of immune cells between the paracancerous and tumor tissues, the characteristics of tumor-infiltrating lymphocytes in HCC tissue can be evaluated (21). Pearson's correlation coefficient analysis and coexpression analysis of these immune cells were performed to determine the interaction of these lymphocytes. In addition, through Kaplan-Meier and Cox multivariate regression analyses, the relationship between TIMH and prognosis was explored. Then, the immune cells associated with 

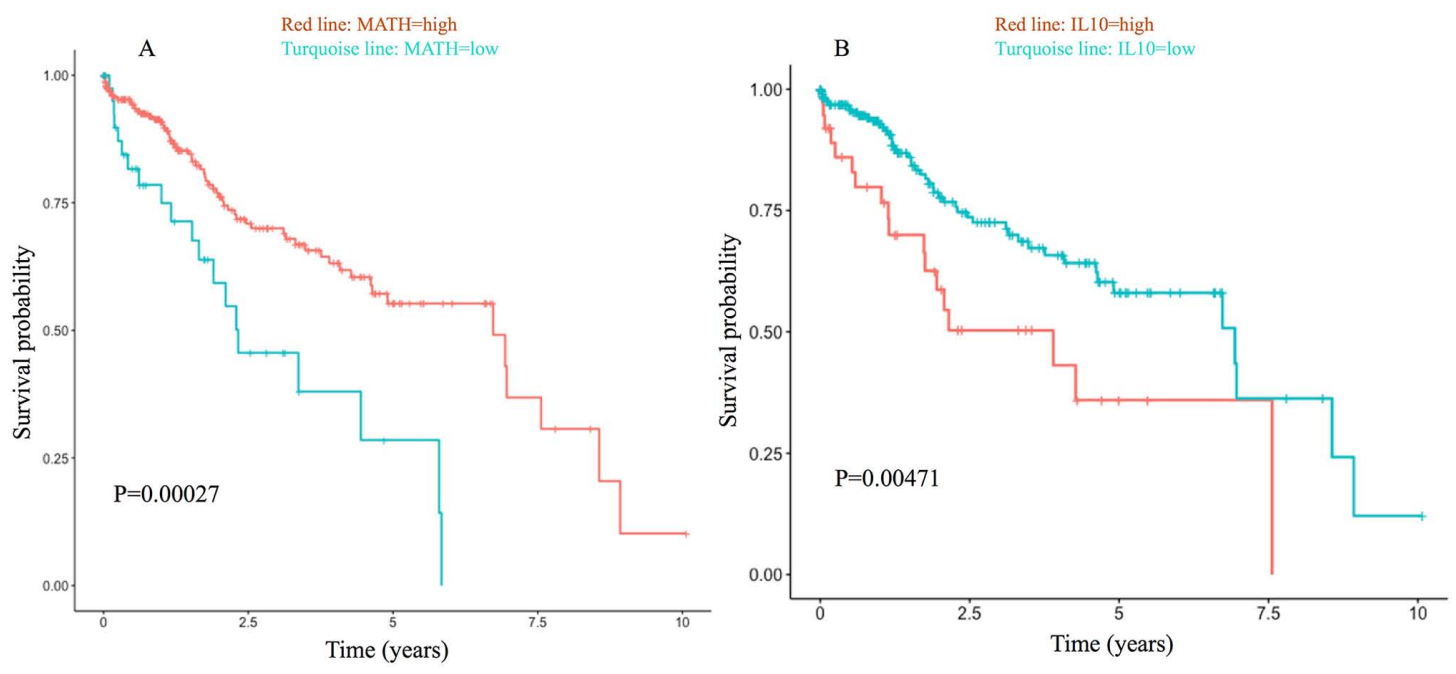

Figure 1. Survival analysis of MATH score and IL10. (A) Survival analysis of OS based on the MATH score. The red line represents the group of high MATH score, and the blue line represents the group of low MATH score, $\mathrm{P}=2.7 \times 10^{-4}$. (B) Survival analysis of OS based on the expression of IL10 in high-score MATH group. $\mathrm{P}=4.71 \times 10^{-3}$. The red line represents the group of high expression of IL10, and the blue line represents the low expression of IL10. OS, overall survival; MATH, mutant-allele tumor heterogeneity; IL10, interleukin 10

overall survival (OS) time were selected for the next step $(\mathrm{P}<0.05)$.

Construction of the immune-related prognostic model. By incorporating IS genes and immune cells into Cox multivariate regression analysis, an immune-related model was established, and the impact of host immunity on OS was further analyzed. In addition, the receiver operating characteristic (ROC) curve and the area under the ROC curve (AUC) were used to evaluate the performance of this model.

The regulatory network of immune-related genes and immune cells. In the aforementioned steps, IS genes were selected by analyzing ITH, and immune-related lymphocytes were chosen by calculating the TIMH. To further investigate the regulatory relationship between these, a regulatory network was established linked by miRNAs and their target genes.

First, the HCC miRNA dataset was downloaded from TCGA, extracted and organized into a miRNA expression matrix. Through univariate linear regression analysis, miRNAs that were highly related to immune cell infiltration were selected $(\mathrm{P}<0.05)$. Second, utilizing miRbase (http://www.mirbase. org), the target genes of these miRNAs were identified, and low-correlation target genes were filtered out. The Database for Annotation, Visualization and Integrated Discovery (DAVID) version 6.8 (https://david.ncifcrf.gov/) was used to analyze the enrichment of the target genes in terms of KEGG pathway and Gene Ontology (GO) annotation and functional classification. The high-correlation target genes and the corresponding miRNAs were used for further analysis.

Finally, the regulatory networks between target genes, miRNAs, immune-related cells and genes were established by Cystoscope software version 3.7.1 (26), and the correlation coefficient map was constructed using R software.

Construction of the network of weighted coexpressed genes and their associations with potential molecules. To further analyze the regulatory mechanisms of these potential molecules (including target genes and miRNAs) in the tumor microenvironment and the signaling pathways involved, the Weighted Correlation Network Analysis Model (WGCNA) (27), a systematic biological method describing the gene correlation pattern between different samples, was constructed. The molecular mechanism, signaling pathways and cell functions were elucidated by analyzing the gene modules corresponding to these potential molecules through WGCNA.

Statistical analysis. Categorized variables were described by proportion (\%) and $\chi^{2}$ tests were used to compare proportions of lymphocytes between normal and tumor tissues. Associations between characteristics and overall survival time were evaluated by Cox proportional hazard models. Kaplan-Meier survival curves and the log-rank test were used to compare overall survival between the high and low MATH score groups. A weighted log-rank test (Renyi test) was performed to generate the P-values when survival curves crossed over. Mantel test was used to measure the correlation between key genes and the matrix of miRNAs and target gene, in which coefficient of correlation (r) described the linear relationship between the two variables. The ratio of lymphocytes between normal and tumor tissue were compared using Wilcoxon rank sum test. The AUC was calculated to assess the predictive ability of the immune-related model.

All statistical analyses were performed using R software (https://www.r-project.org/) and Bioconductor (https://ww w.bioconductor.org/). All statistical tests were two-tailed $\mathrm{P}<0.05$ was consider to indicate a statistically significant difference.

\section{Results}

The MATH score and GSEA. In the present study, the MATH score was a risk factor related to prognosis, and the patients had a poor prognosis in the MATH-low group (MATH score $<19.39$ ) (Fig. 1A). Although the prognosis was improved in the high MATH score group compared with the low MATH 

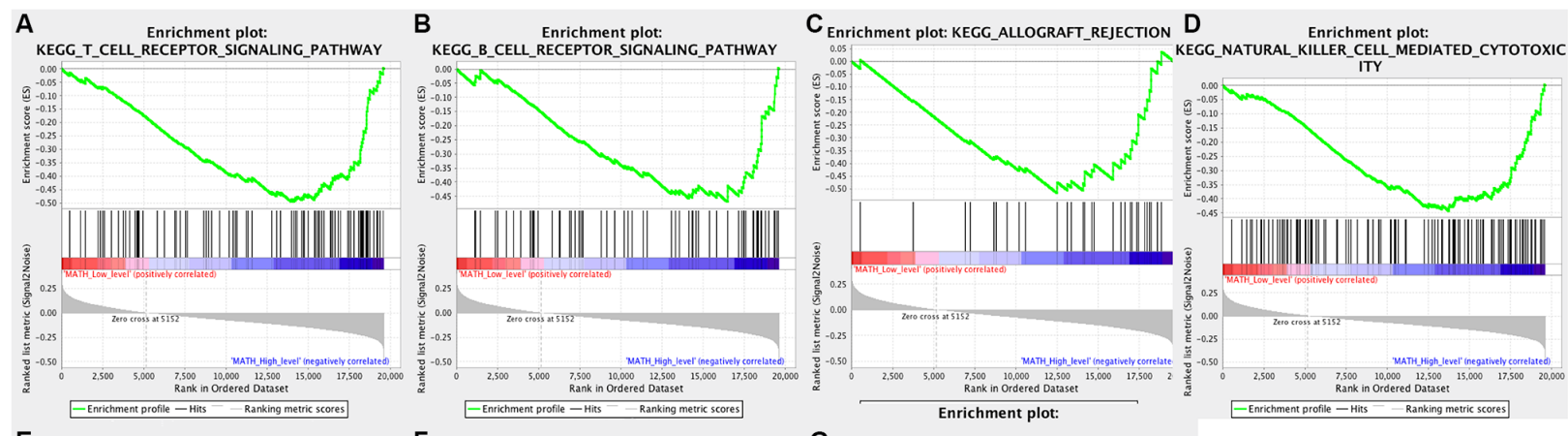

E

$F$

Enrichment plot: KEGG_PRIMARY_IMMUNODEFICIENCY KEGG_ANTIGEN_PROCESSING_AND_PRESENTATION

KEGG_FC_GAMMA_R_MEDIATED_PHAGOCYTOSIS
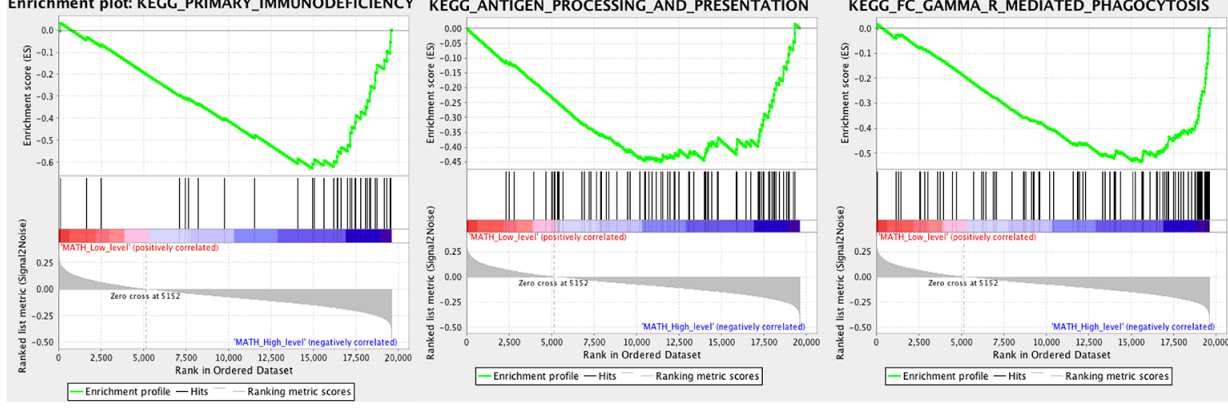

Figure 2. Significant enrichment of the immune-related KEGG pathway in GSEA. (A) KEGG $\mathrm{T}$ cell receptor signaling pathway $\left(\mathrm{NES}=-1.75 ; \mathrm{P}=2.13 \mathrm{x} 10^{-12}\right.$; FDR q-value $\left.=6 \times 10^{-3}\right)$. (B) KEGG B cell receptor signaling pathway $\left(\mathrm{NES}=-1.61 ; \mathrm{P}=1 \times 10^{-3}\right)$. (C) KEGG allograft rejection $\left(\mathrm{NES}=-1.63 ; \mathrm{P}=2.0 \times 10^{-3}\right)$. (D) KEGG natural killer cell mediated cytotoxicity $\left(\mathrm{NES}=-1.59 ; \mathrm{P}=1.46 \times 10^{-19}\right.$; FDR q-value $\left.=2.3 \times 10^{-3}\right)$. (E) KEGG primary immunodeficiency $\left(\mathrm{NES}=-1.96 ; \mathrm{P}=3.19 \times 10^{-21}\right.$; FDR q-value $=1.67 \times 10^{-20}$ ). (F) KEGG antigen processing and presentation (NES=-1.58, $\mathrm{P}=7.3 \times 10^{-21}$; FDR q-value $=2.6 \times 10^{-3}$ ). $(\mathrm{G})$ KEGG FC GAMMA R mediated phagocytosis $\left(\mathrm{NES}=-1.89 ; \mathrm{P}=5.92 \times 10^{-19}\right.$; FDR q-value=2.81 $\left.10^{-18}\right)$. KEGG, Kyoto Encyclopedia of Genes and Genomes; NES, normalized enrichment score; FDR, false discovery rate.

score group (Fig. 1A), the immunosuppression pathways involved, such as 'primary immunodeficiency', are detrimental to prognosis (28). Hence, the immunosuppressive factors in the high-score group were analyzed and it was confirmed that high IL-10 expression was associated with a worse prognosis ( $\mathrm{P}=0.00471$; Fig. 1B). In the GSEA, various gene sets were related to the major nutrients, and the bile acid metabolism gene sets were enriched in the low MATH score group (Table SI). In contrast, seven KEGG signaling pathways associated with immune responses were found in the high MATH score group, including 'allograft rejection', 'B receptor signaling pathway', 'antigen processing and presentation', 'FC gamma R-mediated phagocytosis', 'natural killer cell mediated cytotoxicity', 'primary immunodeficiency' and ' $\mathrm{T}$ cell receptor signaling' (Fig. 2).

Survival and coexpression analysis of immune-related genes. CD4, CD80, interleukin-1 $\alpha$ (IL1A), integrin $\beta-1$ (ITGB2), KRAS and PAK4, which were enriched in these seven immune pathways, were associated with OS. The relationship between these genes and the enriched KEGG pathways is shown in Fig. 3A. ITGB2, IL1A and CD80 were enriched in the primary immunodeficiency pathway. CD4 was enriched in the gene set associated with the primary immunodeficiency pathway, as well as in the $\mathrm{T}$ cell receptor and antigen processing cell-related gene sets.

Coexpression, correlation and cluster analysis for those genes and immune checkpoints were implemented to further study the characteristics of tumor-related immune responses. In the coexpression study, the expression of ITGB2 was positively correlated with PD-L1 and CD4, and the expression of PAK4 was negatively correlated with $\mathrm{C} 8 \mathrm{~A}(\mathrm{P}<0.005$; Fig. 3C-E). The correlation between these immune-related genes and the immune checkpoints ranged from weak to moderate, and they were roughly clustered into four categories by correlation and cluster analysis (Fig. 3B). These immune-related genes may participate in the regulation of tumor immune checkpoints. In the multivariate Cox regression analysis, IL1A and PAK4 were demonstrated to be strong independent risk factors affecting overall survival $(\mathrm{P}=0.00083$ and 0.01257 , respectively; Fig. 4B).

Analysis of immune cells in the tumor immune microenvironment. Considering that the TCGA-LIHC cohort contains only 50 tumor-adjacent tissues, integrated analysis of TCGA data and Genotype-Tissue Expression (GTEx) data was applied to enhance the accuracy of the conclusion. The integrated samples included GTEx normal liver samples and paracancerous samples. Using CIBERSORT, the composition ratio of 22 immune cell types in the HCC samples was calculated. Accordingly, the difference in the infiltrating immune cell types between integrated samples and tumor tissue was estimated. In each tumor tissue, the proportion of different immune cells varied, indicating TIMH. The proportion of M0 macrophages was higher in the tumor tissue compared with integrated samples, and the proportion of M2 macrophages was lower in the tumor tissue compared with in the integrated samples (Fig. 5A).

The correlation matrix of all 22 lymphocyte types showed their correlation from weak to moderate (Fig. 5B). Univariate and multivariate Cox analyses of these 22 immune cell types were employed to analyze the relationship between TIMH and 
A

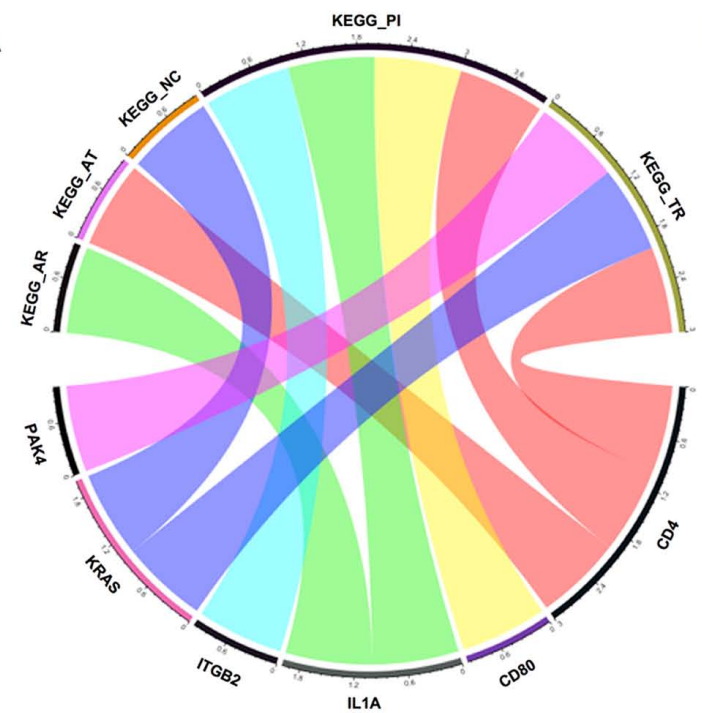

B

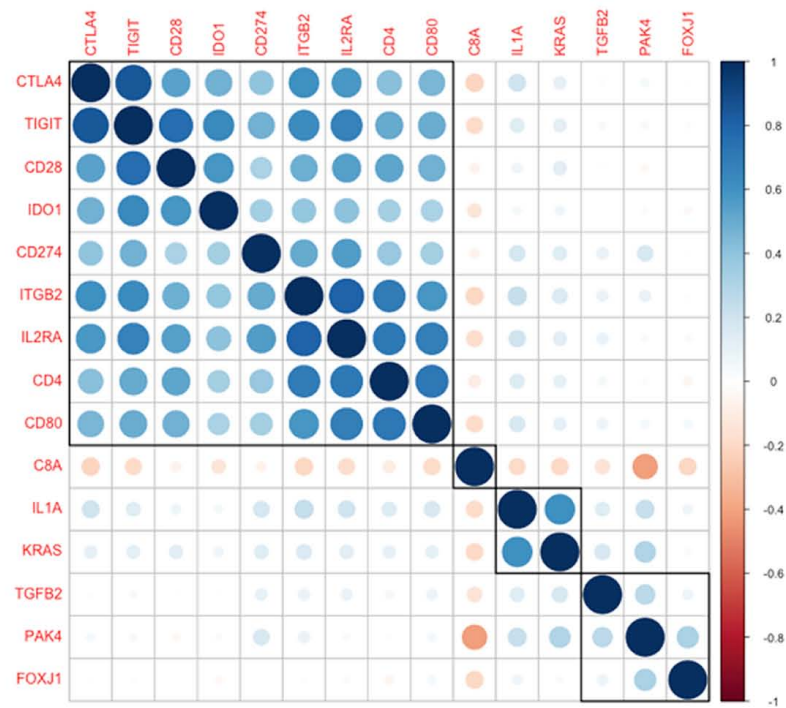

E Cor $=0.469\left(p-v a l u e=6.922^{*} 10^{-22}\right)$

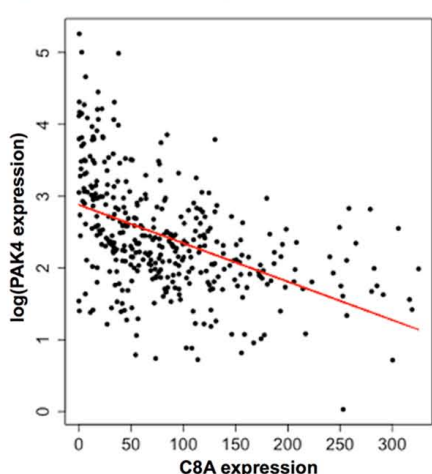

Figure 3. KEGG pathway, correlation and co-expression analysis of immune genes. (A) Circular plot of immune related pathways enriched for the immune genes. (B) Correlation matrix of all immune-related genes and immune checkpoints, and correlation varies from weak to strong. (C-E) Co-expression analysis between immune related genes and immune checkpoints. (C) Co-expression analysis of ITGB2 and CD274 (coefficient $=0.568 ; \mathrm{P}=1.98 \times 10^{-25}$ ). (D) Co-expression analysis of ITGB2 and CD4 (coefficient=0.599; P=5.39x10-29). (E) Co-expression analysis of C8A and PAK4 (coefficient=0.469; P=2.53x10 ${ }^{-19}$ ). ITGB2; integrin $\beta-1$; PAK4, serine/threonine p21-activated kinase 4; KEGG, Kyoto Encyclopedia of Genes and Genomes.
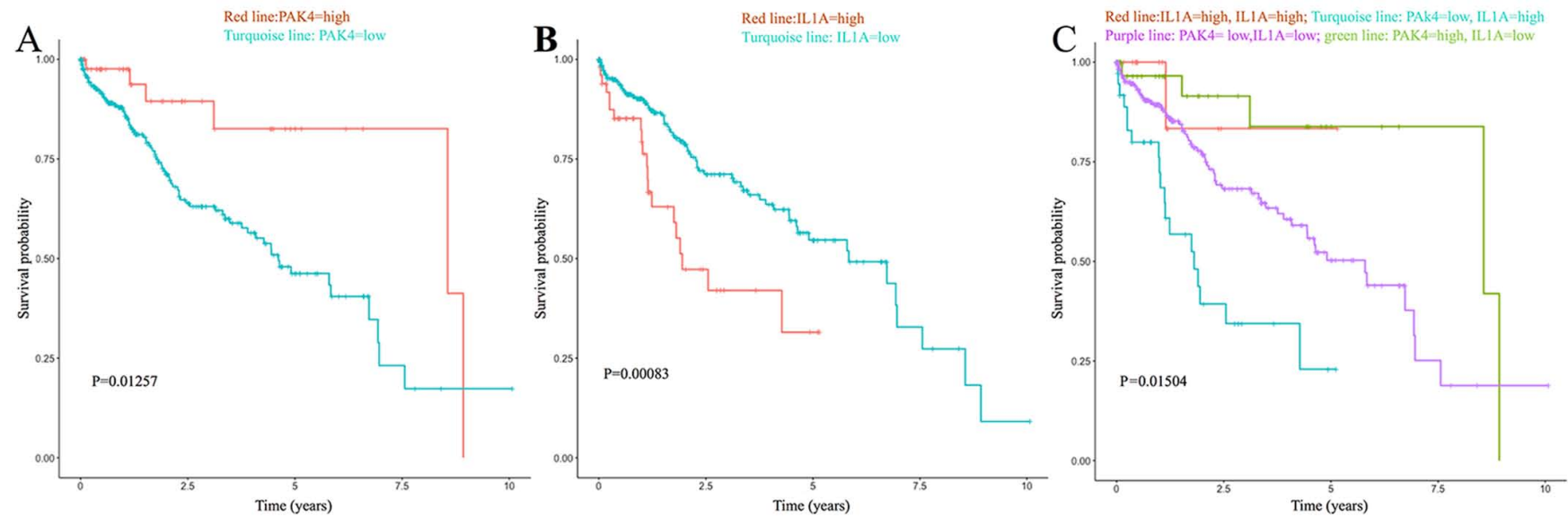

Figure 4. Survival analysis of PAK4 and IL1A. (A) The survival analysis of the expression of PAK4. The red line represents high expression of PAK4, and the blue line represents the low expression of PAK4. (B) The survival analysis of the expression of IL1A. The red line represents high expression of IL1A, and the blue line represents low expression of IL1A. (C) The survival analysis of the expression of PAK4 and IL1A. The red line represents high expression of PAK4 and IL1A. The purple line represents low expression of PAK4 and low expression of IL1A. The green line represents low expression of PAK4 and high expression of IL1A, and the blue line represents low expression of PAK4 and high expression of IL1A. PAK4, serine/threonine p21-activated kinase 4; IL1A, interleukin 1- $\alpha$.

prognosis. Based on the survival analyses of these immune cells, OS time was extended when the proportion of M1 macrophages was higher but shortened if the proportion of M2 macrophages was higher in the tumor tissues. Then, the 
A

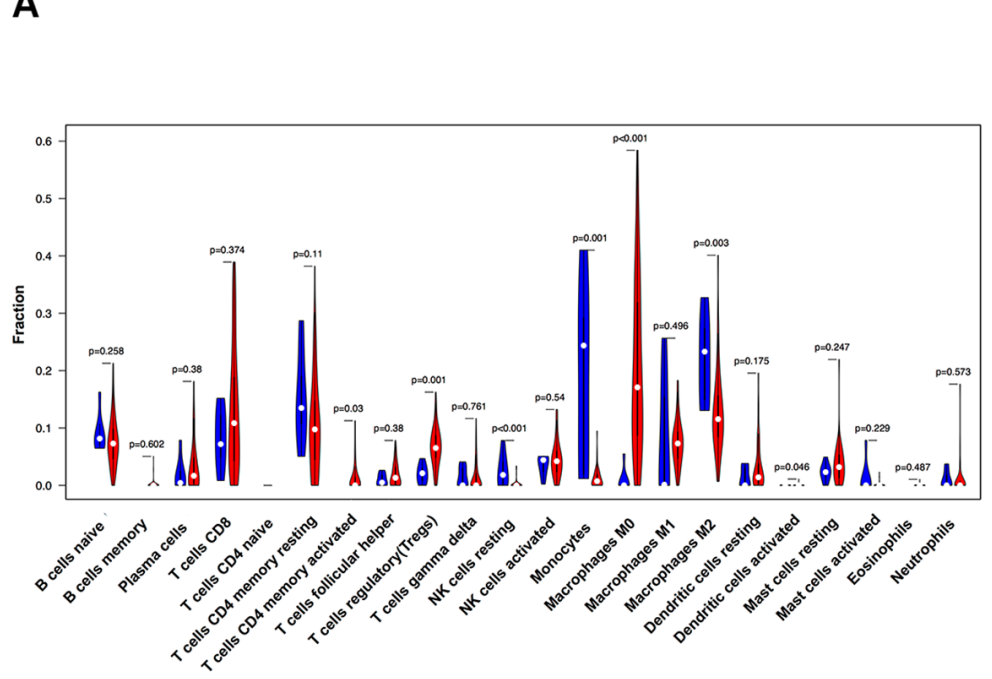

B

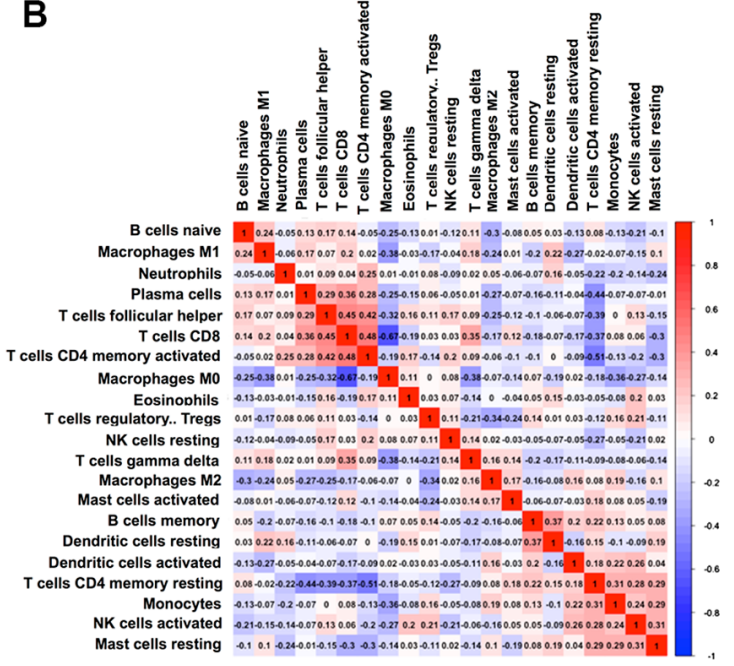

Figure 5. Violin plot and correlation map of immune-infiltrating lymphocytes. (A) Violin plot of immune-infiltrating lymphocytes between tumor tissue and integrated samples (paracancer and healthy liver tissues), in which the red represents tumor samples, and the blue represents integrated samples. (B) The correlation matrix of all 22 lymphocytes. Their correlation varies from weak to strong. NK, natural killer.

phenotype of macrophage polarization was assessed with the ratio of $\mathrm{M} 1 / \mathrm{M} 2$ set as the biomarker (the cut-off value was set as 0.7284), and the results showed that the ratio was better at predicting survival compared with the proportion of $\mathrm{M} 1$ or M2 macrophages alone (Fig. 6A, B and F). The percentages of NK-activated cells and Tregs were 4.84 and $6.26 \%$, respectively, among all lymphocytes. In addition, a high proportion of NK activated cells and regulatory $\mathrm{T}$ cells worsened the prognosis of HCC (Fig. 6C and D). Thus, in the Cox multivariate analysis, the M1/M2 ratio was confirmed as a strong independent risk factor for prognosis, and OS was prolonged when the proportion was above $72.83 \%$ (Fig. 6F).

Establishing the immune-related prognosis model. Furthermore, the immune-related prognosis model in the present study was used to indirectly investigate the effects of ITH and TIMH on prognosis after integrating IL1A, PAK4 and $\mathrm{M} 1 / \mathrm{M} 2$ ratio into the Cox multivariate regression analysis (Fig. 6E and F). After forward stepwise regression, the M1/M2 ratio and IL1A were retained in the model. The model yielded an AUC of 0.7 in the prediction of 3-year survival, which was higher compared with that of the IL1A/PAK4 model $(\mathrm{AUC}=0.6)$ and the $\mathrm{M} 1 / \mathrm{M} 2$ ratio model $(\mathrm{AUC}=0.651)$. In the present model, the ability to predict 5-year survival was even better, with a higher AUC (0.9) (Fig. 7) compared with the IL1A/PAK4 model and the M1/M2 ratio model.

The interaction and regulation between ITH and TIMH. The link between the M1/M2 ratio and immune-related genes was investigated through examining miRNAs and their corresponding target genes to study the molecular regulation mechanisms of macrophage polarization and establish a regulatory network between IL1A, PAK4 and the M1/M2 ratio. The miRNA expression matrix was extracted and subjected to regression analysis with the M1/M2 ratio. In the present study, 19 related miRNAs were obtained. After excluding the miRNAs with very low expression levels, the eligible miRNAs that remained were miRNA-191, -6798, -1269 and -4661 . Then,
1,174 target genes of these miRNAs were identified using the miRBase database, and a target gene expression matrix was established. In the gene expression matrix, 92 target genes had a strong linear relationship with the M1/M2 ratio, and KEGG enrichment analysis of these 92 target genes was performed using the DAVID dataset. The main enriched KEGG pathways were as follows: 'MicroRNAs in cancer'; 'thyroid hormone signaling'; 'renal cell carcinoma'; 'tight junctions'; 'herpes simplex infection' and 'ErbB signaling' (Table SII).

Among the microRNAs in the cancer pathway, PAK4 and CAT-1 participate in the transformation of normal hepatocytes to HCC. In the ErbB signaling pathway, PAK4 and JNK participate in tumor angiogenesis, which is associated with macrophage polarization (29). Inhibitor of nuclear factor $\kappa$-B kinase (IKKB), inhibitor of nuclear factor $\kappa$-B kinase $\alpha$ (IKKA) and interferon $\alpha-2$ are enriched in the 'herpes simplex infection pathway', which suggests that these genes promote macrophage polarization. In addition, ZO-1 and tight junction-associated protein 1 were enriched in the 'cell polarity', and ZO-1 and ZONAB were enriched in the 'cell differentiation' and 'reduced cell proliferation' (Table SII). These KEGG pathways may be related to macrophage polarization. In the 92 target genes, seven genes linear to their corresponding miRNAs were selected as follows: Angiotensin II type 1 receptor (AGTR1), calbindin (CALB1), double cortin domain-containing protein 1 (DCDC1), glycosaminoglycan xylosylkinase (FAM20B), neuromodulin (GAP43), heat shock protein $105 \mathrm{kDa}(\mathrm{HSPH} 1)$ and serine/threonine p21-activated kinases 4 (PAK4). Finally, the correlation matrix map of the seven target genes and four miRNAs linked with the M1/M2 ratio, IL1A and PAK4 was plotted to show their correlations, which ranged from weak to strong. The relationship between $\mathrm{M} 1 / \mathrm{M} 2$ ratio, IL1A, PAK4, miRNAs and the target genes is shown in Fig. 8A.

In the final modulation network (Fig. 8B), three pairs of miRNAs and target genes with linear correlations were obtained as follows: MiRNA-1269b with GAP43; miR-4661 with HSPH1 and miRNA-6798 with PAK4. In addition, PAK4 


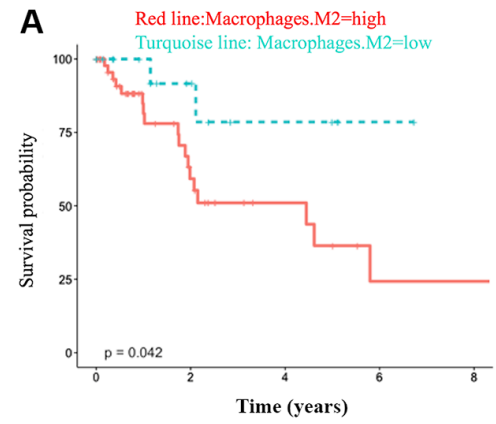

C

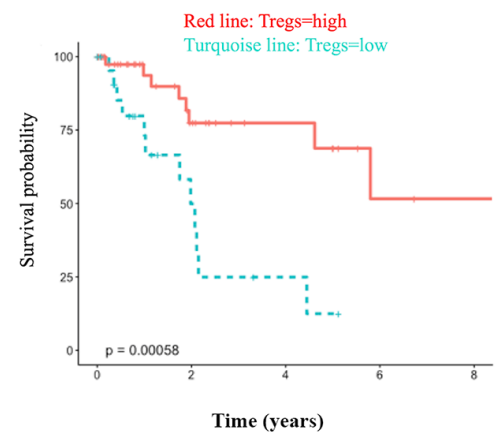

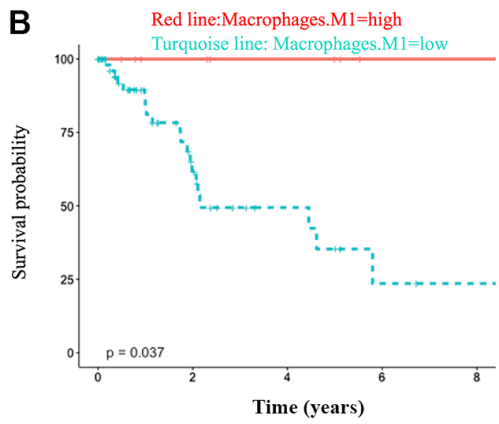

D

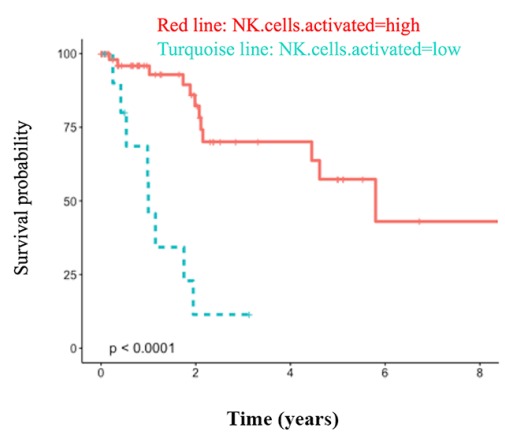

E
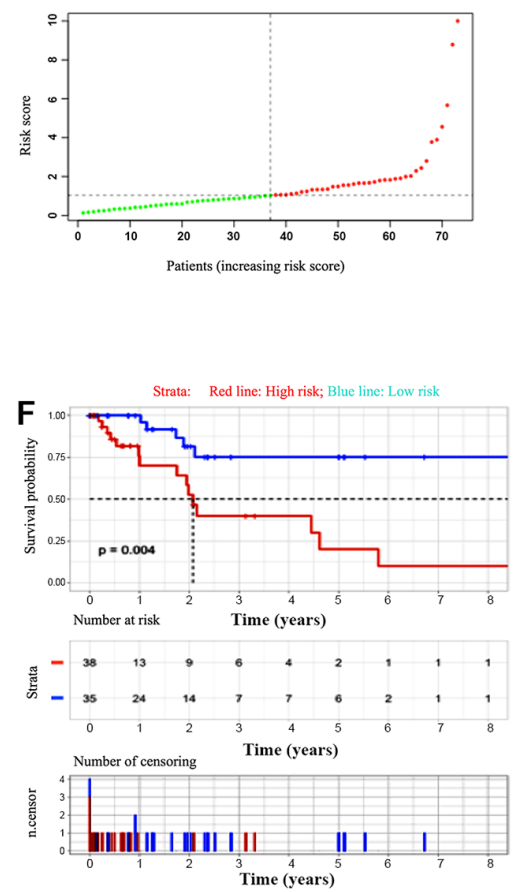

Figure 6. Survival analysis of M1, M2 macrophages and the immune model. (A-D) The survival analysis of different proportion of M1 macrophages, M2 macrophages, activated NK cell and Tregs. The red line represents high proportion of those lymphocytes and the blue line represents low proportion of those lymphocytes (A) $\mathrm{P}=0.042$, (B) $\mathrm{P}=0.037$, (C) $\mathrm{P}=0.00058$ and (D) $\mathrm{P}<0.0001$ ). (E) Risk score distribution of the M1/M2 ratio and IL1A signature. (F) Kaplan-Meier analysis of the immune model (M1/M2 ratio and IL1A). The red line represents high risk score and the blue line represents low risk score, $\left.\mathrm{P}=4.0 \times 10^{-3}\right)$. NK, natural killer; Tregs, regulatory T cells; IL1A, interleukin 1- $\alpha$.
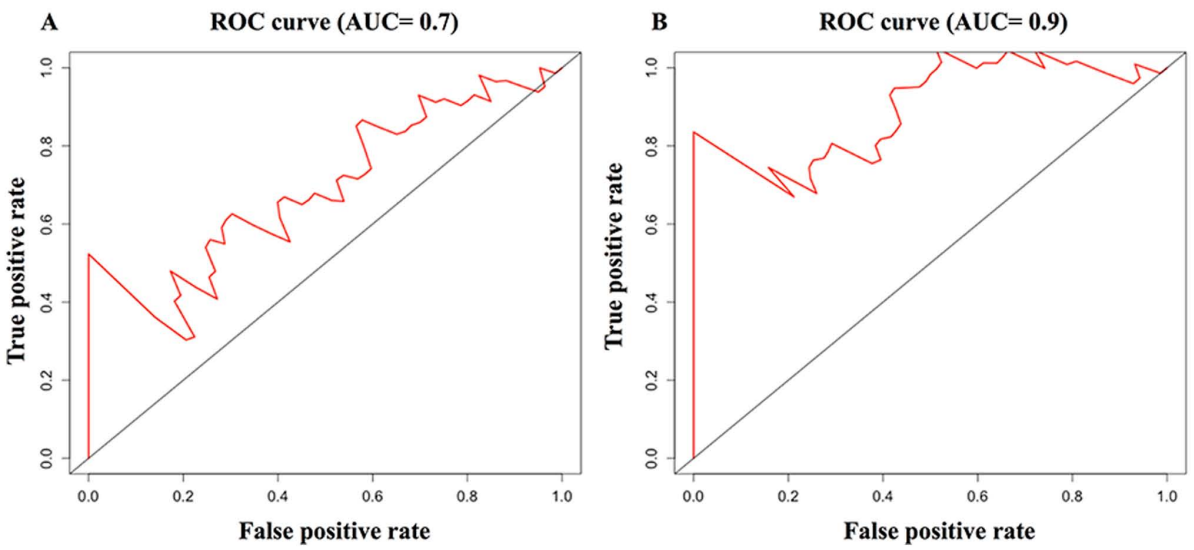

Figure 7. Survival risk prediction of immune-related model in (A) 3-years and (B) 5-years. ROC, receiver operating characteristic; AUC, area under the curve.

is not only an immune-related gene but is also a target gene of miRNA-6798. In the regulation network and correlation coefficient map, it was demonstrated that miRNA-6798 positively regulates the polarization of macrophages and governs the expression of PAK4. IL1A is coexpressed with CALB1, GAP43 and HSPH1. Therefore, IL1A and PAK4 indirectly govern the differentiation of macrophages by coexpression with HSPH1. Finally, coexpression analysis was applied again to verify the linear correlation between the expression of PAK4, HSPH1 and MATH scores (correlation value of PAK4=0.268; $\mathrm{P}<0.05$ ), which may indicate the key role of PAK4 in tumor cell differentiation (Fig. S1).

Then, all these coexpressed genes were analyzed with respect to the expression of those molecules. The yellow consensus module showed the most significant correlation with the expression of PAK4 (correlation value $=0.8$; $\mathrm{P}<0.001$; Fig. 9C). The brown consensus module showed the most significant correlation with the expression of HSPH1 (correlation value $=0.51 ; \mathrm{P}<0.001$; Fig. 9C). The turquoise consensus module showed the most significant correlation with the expression of miRNA-1911 (correlation value $=0.39 ; \mathrm{P}<0.001 ;$ Fig. $9 \mathrm{C}$ ). $\mathrm{GO}$ analysis indicated enrichment of mRNA processing in gene sets related to PAK4, cell-substrate adhesion, cell-substrate junction and cell adhesion binding in gene sets related to HSPH1, and chromosome segregation, nuclear division and nuclear chromosome segregation in gene sets related to miRNA-1911 (Figs. 10C, 11C and 12C, respectively). KEGG pathway 

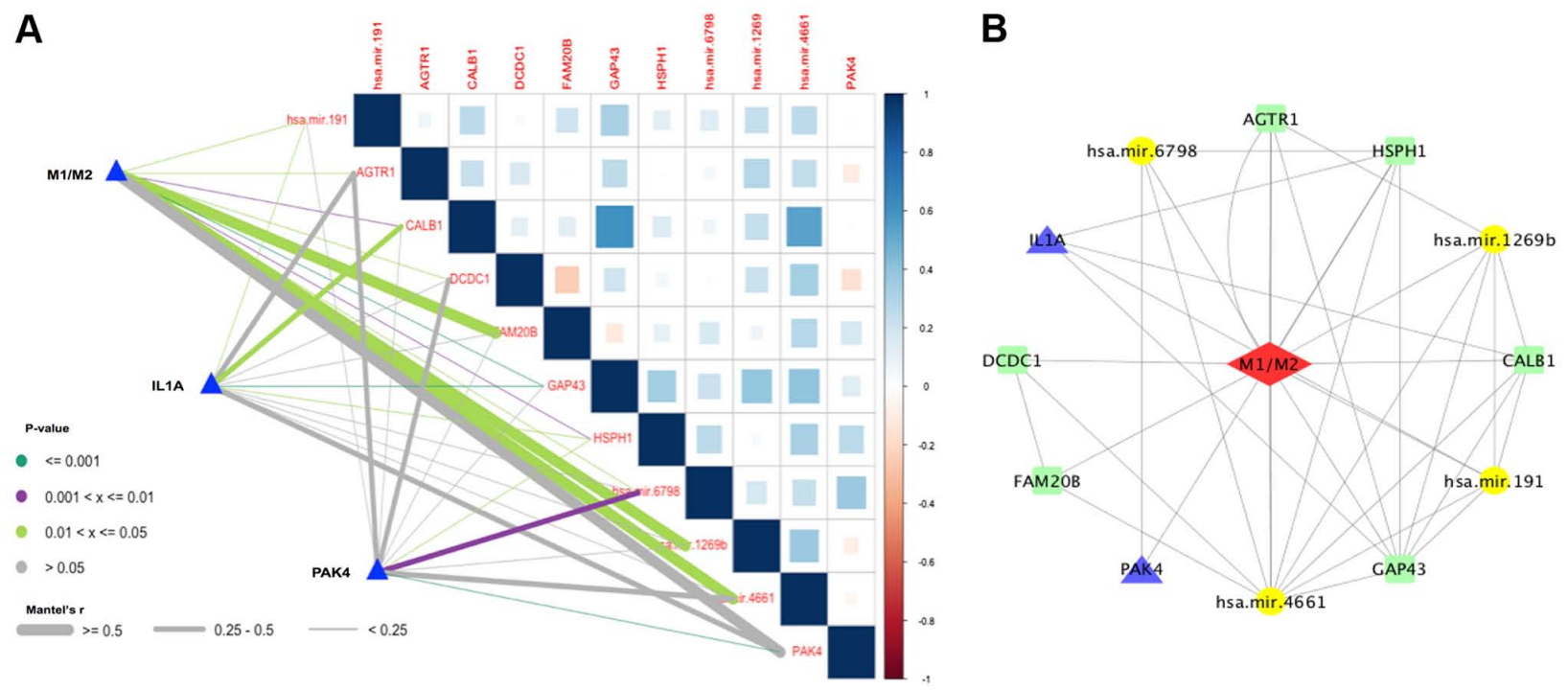

Figure 8. Map of correlation and regulatory network between biomarkers. (A) The relationships between M1/M2 ratio, IL1A, PAK4, miRNAs and target genes. Mantel test was applied using 'vegan' $R$ package. Mantel's $r$ (coefficient of correlation) represents the correlation, in which thickness of the line represents the level of correlation and the color of the lines represents the P-value. (B) The regulatory topology network of immune-related genes, M1/M2 ratio, miRNAs and target genes. Blue triangles represent key genes (IL1A and PAK4); yellow circles represent miRNAs; green squares represent target genes; the parallelogram represents M1/M2 ratio and lines illustrates their linear relationship. IL1A, interleukin 1- $\alpha$; PAK4, serine/threonine p21-activated kinases 4; miRNA, microRNA.

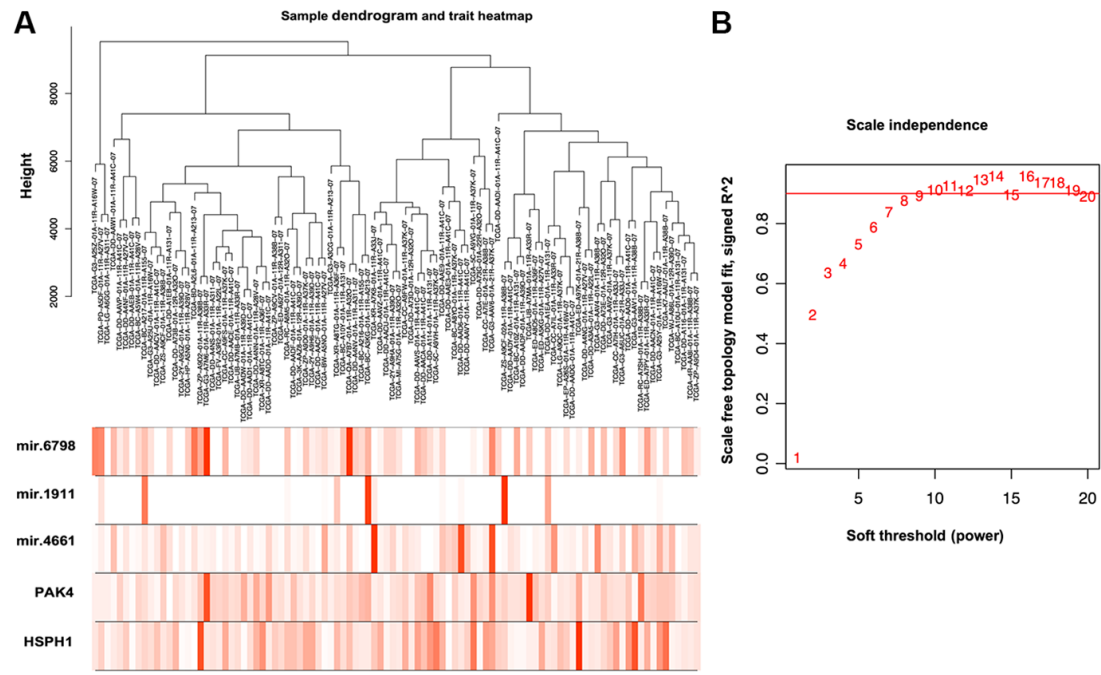

C

Figure 9. Network construction of the weighted co-expressed genes and their associations with potential molecules (PAK4, HSPH1 and miRNA-1911). (A) Dendrogram produced by average linkage hierarchical clustering of the identified co-expression modules. (B) Scale free topology model fit. (C) Module-trait relationships between the identified modules and potential molecules. The numbers represent Pearson's correlation between the clinical traits and modules. The numbers in the parentheses correspond to the P-values. The background colors of the numbers represent the strength of the correlation. PAK4, serine/threonine p21-activated kinase 4; HSPH1, heat shock protein $105 \mathrm{kDa}$; miRNA, microRNA.

analysis showed the association of 'herpes simplex virus 1 infection' with PAK4; 'human papillomavirus infection' with HSPH1 and the 'Fanconi anemia pathway' with miRNA-1911 (Figs. 10B, 11B and 12B).

\section{Discussion}

The occurrence of non-synonymous mutations in HCC is a condition for the production of new antigens that have not previously been detected by the immune system (30). ITH stems from the production of neoantigens, which can induce immune surveillance and immune responses (31). Moreover, the development of HCC is thought to be associated with the immune response and the immune microenvironment (18). The immune microenvironment is often accompanied by chronic liver inflammation in the occurrence of HCC. The differences in immune cells, immunoregulatory factors and their gene and protein profiles in the microenvironment of liver cancer reflect the heterogeneity of the tumor immune microenvironment and are associated with prognosis, immune response and drug resistance (22). The present study applied the MATH algorithm and CIBERSORT method to evaluate the ITH and TMH. By evaluating the MATH score, it was reported that there was no difference in OS when grouped by the median score. However, when using 19.39 as the optimal cutoff value, the overall survival in the low MATH score group was lower 
A

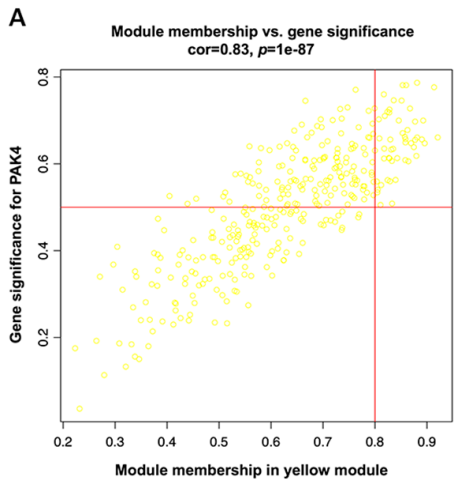

B

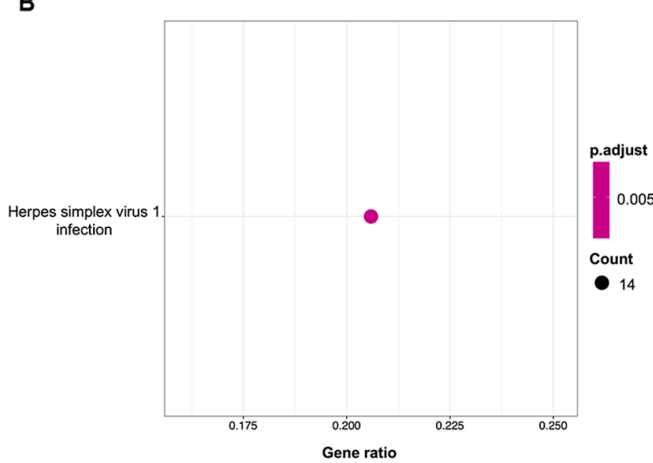

C

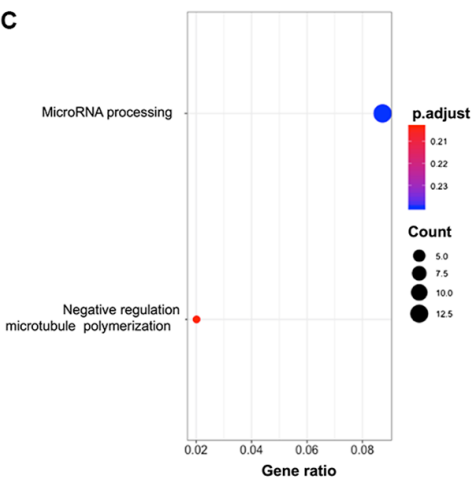

Figure 10. Scatterplots of PAK4 and KEGG and GO analysis. (A) Scatterplots of gene significance for the expression of PAK4. (B) Bubble chart of the enrichment Kyoto Encyclopedia of Genes and Genomes pathways for the gene sets of PAK4. The cut-off criterion was P $<0.05$. (C) Bubble chart of significant Gene Ontology terms of the gene sets of PAK4. The cut-off criterion was $\mathrm{P}<0.05$. PAK4, serine/threonine p21-activated kinase 4.
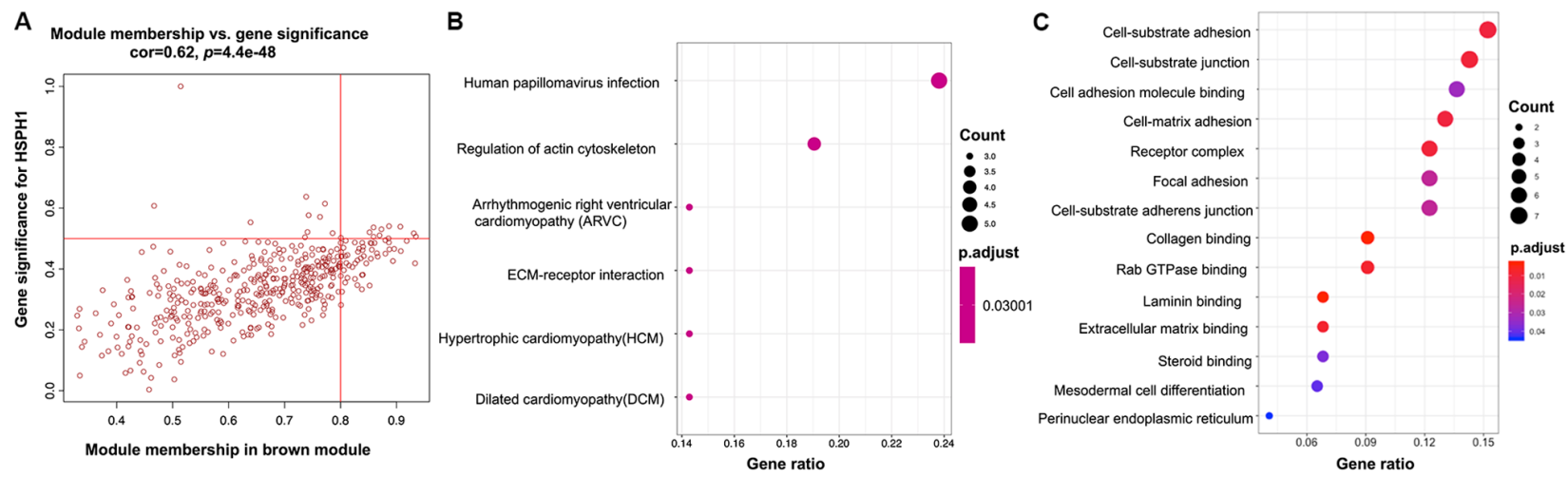

Figure 11. Scatterplots of HSPH1, KEGG and GO analysis. (A) Scatterplots of gene significance for the expression of HSPH1. (B) Bubble chart of the enrichment KEGG pathways for the gene sets of HSPH1. The cut-off criterion was $\mathrm{P}<0.05$. (C) Bubble chart of significant GO terms of the gene sets of HSPH1. The cut-off criterion was P<0.05. HSPH1, heat shock protein 105 kDa; KEGG, Kyoto Encyclopedia of Genes and Genome; GO, Gene Ontology.
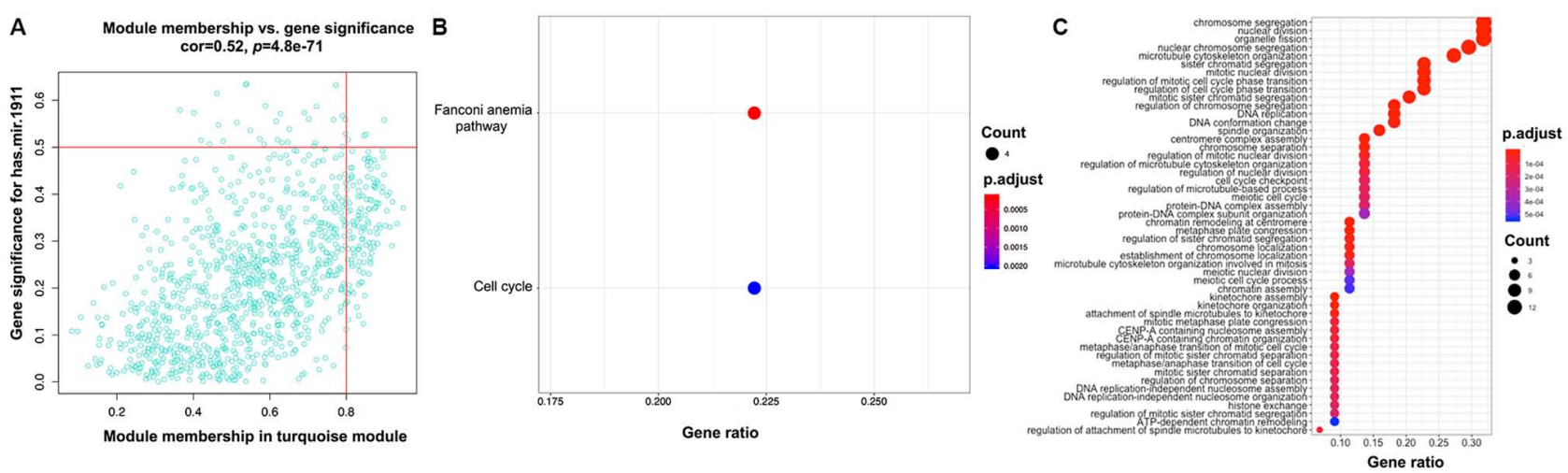

Figure 12. Scatterplots of miRNA-1911, KEGG and GO analysis. (A) Scatterplots of gene significance for the expression of miRNA-1911. (B) Bubble chart of the enrichment Kyoto Encyclopedia of Genes and Genomes pathways for the gene sets of miRNA-1911. The cut-off criterion was $\mathrm{P}<0.05$. (C) Bubble chart of significant Gene Ontology terms of the gene sets miRNA-1911. The cut-off criterion was $\mathrm{P}<0.05$. miRNA, microRNA; KEGG, Kyoto Encyclopedia of Genes and Genome; GO, Gene Ontology.

compared with that of the high MATH score group, which was different from previous reports which demonstrated that high MATH scores indicate poor prognosis in colon cancer and diffuse large B-cell lymphoma $(15,16)$. The low MATH score group accounted for only $13 \%$ of the cohort; however, it is still necessary to resolve the reasons for the differences observed.

In the GSEA, three major nutrient metabolism pathways and the bile acid metabolism pathway were enriched in the low MATH score group, while seven immune-related gene sets were enriched in the high MATH score group. Therefore, it can be concluded that extremely low ITH indicates lower tumor immunogenicity, which protects tumor cells from being recognized by the immune system and avoids the activation of anticancer immunity (30). This also explains the poor prognosis phenomenon in the low MATH score group. However, compared with the overall survival of the total study cohort, 
there was no difference in the overall survival of the high MATH score group. As shown in the results, the enriched immunosuppressive pathway may influence prognosis in the high MATH score group. The overexpression of the immunosuppressive factor IL10 indicated shortened survival time in the high MATH score group. The aforementioned results indicated the paradoxical role of cytokines in tumor immunity. The tumor immune response is highly related to a complex regulatory process of ITH $(32,33)$.

Next, the genes enriched in the immune-related pathway were identified in the high MATH score group and confirmed to be associated with OS. ITGB2 was positively correlated with CD4 and PD-L1, and when ITGB2 was upregulated, the survival time is shortened. The correlation between these genes and immune checkpoints ranged from weak to moderate. IL1A and PAK4 were also found to be strongly independent risk factors for survival through multivariate analysis. IL1A was enriched in the 'primary immunodeficiency' and 'allograft rejection' pathways, and PAK4 was also enriched in the 'T cell receptor signaling pathway'. The IL1A cytokine is produced by macrophages and monocytes and is involved in various immune responses and inflammatory processes, such as stimulated the production of chemokines resulting in the infiltration of neutrophils (34). IL1A affects various stages of carcinogenesis, tumor growth and tumor cell invasiveness and also the pattern of interactions between tumor cells, the host immune system and the immune microenvironment, in which IL-1 may also enhance the invasiveness of tumor cells by the induction of heparanase, chemokines or integrins on the malignant cells or endothelial cells (35). PAK4 is upregulated in tumor tissue, especially pancreatic cancer and oral squamous cell carcinoma. The amplification of PAK4 plays an important role in tumor invasion associated with poor prognosis (36). It has been reported that the growth of breast cancer is suppressed when the PAK4 pathway is inhibited in in vitro experiments (37). In addition, miRNA-199a-regulated PAK4 promotes HCC occurrence, and PAK4-regulated TP53 promotes HCC progression in in vivo experiments (38). In the $\mathrm{T}$ cell receptor signaling pathway, PAK4 acts as an inhibitor of the regulation of the actin cytoskeleton and effectively protects $\mathrm{T}$ cells from the host immune response (39).

The CIBERSORT algorithm was employed to determine the percentage of lymphocytes in a bulk of tumor transcriptomes to explore the tumor immune mechanism and TIMH from leukocyte infiltration in the HCC immune microenvironment. Previous studies have conducted a similar analysis of immune cell infiltration in HCC tissues and the impact of immune cells, such as macrophages and Tregs on prognosis $(40,41)$. One of the studies concluded that a higher ratio of M1 macrophages indicates an improved prognosis (40), while another study illustrates that the total number of macrophages was negatively correlated with OS (41). To gain more insight into the relationship between the ratio of different types of macrophages and prognosis, the present study analyzed the relationship between the content of three types of macrophages and prognosis separately and also clarified the effect of the $\mathrm{M} 1 / \mathrm{M} 2$ ratio on prognosis. The percentage of M0 macrophages in tumor tissues was higher compared with that in normal tissues. Tumor-associated macrophages are important components of the HCC immune microenvironment (42). In the present study, among the 22 types of infiltrating lymphocyte, M0, M1 and M2 accounted for $20.05,7.76$ and $13.24 \%$, respectively. In the HCC microenvironment, M2 macrophages are the characteristic phenotype of tumor-associated macrophages, which promote angiogenesis to support tumor cell invasion and metastasis $(43,44)$. Based on previous research, M2 macrophages are associated with poor clinical prognosis, while M1 macrophages are considered to inhibit tumors growth and are tumoricidal (45). However, studies have also shown that M1 macrophages can induce epithelial stromal cell transformation of pancreatic ductal adenocarcinoma (46), activate hepatoma cells (47) and induce PD-L1 expression (42). In the present study, the survival time was prolonged when the M1 percentage was high, while the survival time was shortened when the M2 percentage was high. The cut-off value for the M1/M2 ratio was set as 0.7284 , and the HCC cohort was divided into high-risk and a low-risk groups. The survival of the two groups was significantly different $(\mathrm{P}=0.004)$, which further indicated that the polarization of macrophages into M1 can bring about good prognosis, while M2 usually predicts a poor prognosis. The balance of M1-M2 macrophages is related to various cancer and inflammatory diseases, such as melanoma, lung cancer and asthma (48-50), and the ratio of $\mathrm{M} 1 / \mathrm{M} 2$ also serves as a risk factor for survival in the present study. Furthermore, the proportion of NK-activated cells and Tregs cells was also associated with clinical prognosis. The percentages of NK-activated cells and Tregs were 4.84 and $6.26 \%$, respectively, among all lymphocytes. This was consistent with a previous study that demonstrated that tumor-infiltrating NK-activated cells play a role in immune surveillance and killing tumor cells by natural cytotoxicity (51). In multivariate Cox regression analysis, the M1/M2 ratio acted as the best biomarker for survival prediction. Next, IL1A and the M1/M2 ratio were integrated into the final model by multivariate Cox regression analysis. The predictive ability of the model was judged by the AUC value, wherein the 3-year prediction accuracy was 0.651 and the 5-year prediction accuracy was higher with an average value of 0.9 . Moreover, the heat map of the risk score for immune cells and the gene model showed that the ratio of the M1/M2 ratio was notably different in the high- and low-risk groups compared with the IL1A group. Therefore, the $\mathrm{M} 1 / \mathrm{M} 2$ ratio is a critical factor in the model risk score.

In a previous study, macrophage polarization modulated by miRNAs has been studied (48). In the present study, the expression of miRNA-1269b, $-6798,-191$ and -4661 was linearly related to the $\mathrm{M} 1 / \mathrm{M} 2$ ratio, indicating that these miRNAs affect macrophage polarization. It has also been demonstrated that miRNA-4661 can regulate the immune response through the expression of IL10 (52), and miRNA-191 is highly expressed in HCC and involved in promoting the cell cycle and tumor cell proliferation (53). Activation of the $\mathrm{NF}-\kappa \mathrm{B}$ pathway promotes the expression of miRNA-1269b, inducing the development of liver cancer (54). The seven target genes (AGTR1, HSPH1, CALB1, GAP43, FAM2OB, $D C D C 1$ and $P A K 4)$ were also related to the M1/M2 ratio. Furthermore, IL1A was highly correlated with HSPH1, $C A L B 1$, miRNA-191 and GAP43. PAK4 was highly correlated with HSPH1. The relationship network of these miRNAs, 
target genes, immune-related genes and the M1/M2 ratio underpin the regulatory relationship between IL1A and PAK4 and the M1/M2 ratio. As shown in the results, both IL1A and PAK4 regulate macrophage polarization through HSPH1, which may indicate that HSPH1 is an essential factor in macrophage polarization regulation and in the immune microenvironment of liver cancer. In the colon cancer immune response, HSPH1 modulates macrophage polarization (55) and is associated with chemotherapy sensitivity (56) and immunogenicity (57). The present study also indicated that HSPH1 may play a role in regulating macrophage polarization and tumor immune response in liver cancer. By establishing the regulatory network of IL1A, PAK4, HSPH1 and $\mathrm{M} 1 / \mathrm{M} 2$ ratio, it was clarified how the tumor immune response mediated by ITH alters tumor-infiltrating immune cells and thus affects TIMH in HCC. On the other hand, PAK4 not only plays a regulatory role in the development of HCC but also plays a marked role in mutation, neoantigen production and regulation of tumor immunity. Moreover, HSPH1 is a mediator in the network that regulates ITH and TIMH in HCC. In the WGCNA, the gene sets were enriched in 'herpes simplex virus 1 infection', 'human papillomavirus infection' and 'Fanconi anemia', which were in turn related to immune responses and macrophage differentiation (58) illustrating the potential of these molecules to regulate the tumor immune microenvironment. It has been reported that DNA-dependent protein kinase/Akt-mTORC1, Toll-like receptor/nitric oxide and DNA-dependent protein kinase (DNA-PK) / Rac1 signaling pathways are associated with the immune-related KEGG pathway, therefore these may have a role in the altered microenvironment of HCC (59-61).

Overall, the present study explored the potential relationship between HCC ITH and TIMH via bioinformatics analysis. According to the multi-omics analysis, ILIA, $P A K 4$ and $H S P H 1$ may be key genes in tumor evolution, and liver cancer immune-related signaling pathways were identified. Furthermore, miRNA-1269b, -6798, -191 and -4661 are involved in the regulation of the tumor microenvironment and may play an important role in cross-talk between tumor cells and immune cells. Owing to the experimental limitation, the majority of the present study focused on multi-omics analysis of bioinformatics, whereas discussion on other tumor immune microenvironment factors, such as fibroblasts, transforming growth factors, chemokines were not included. Considering that the immune microenvironment of liver cancer is extremely complex, further exploration is needed in future studies, such as the effect of glycolytic metabolites on the polarization of macrophages and the molecular interaction mechanism between immune cells and tumor cells.

\section{Acknowledgements}

Not applicable.

\section{Funding}

This work was funded by a Grant-In-Aid for Scientific Research from the National Natural Science Foundation for the Youth of China (grant no. 81704042).

\section{Availability of data and materials}

The datasets generated and/or analyzed during the current study are available in The Cancer Genome Atlas repository, (https://portal.gdc.cancer.gov/) and Genotype-Tissue Expression repository (https://xenabrowser.net/datapages).

\section{Authors' contributions}

LBL designed and conceived the study. LY collected the MAF files in the TCGA-LIHC database and calculated the MATH score to analyzed the association between MATH score and survival time. GQX collected the RNA seq files to explore the oncological features of immune genes via GSEA and KEGG pathways. XCZ collected and integrate analysis Genotype Tissue Expression with TCGA dataset to analyze the difference in the percentage of immune cells by CIBERSORT algorithm. QLX collected and analyzed TCGA-LIHC miRNA dataset to establish the regulatory networks between target genes and miRNAs. XBS organizes TCGA RNA-seq and miRNA dataset, and analyzes the correlation of immune-related genes and draws relevant figures. XDG made substantial contributions to conception and design of this study and participated in drafting the manuscript. ZYM polished the language and performed the data analyses. All authors read and approved the final version of the manuscript.

\section{Ethics approval and consent to participate}

Not applicable.

\section{Patient consent for publication}

Not applicable.

\section{Competing interests}

The authors declare that they have no competing interests.

\section{References}

1. Nault JC, Ningarhari M, Rebouissou S and Zucman-Rossi J: The role of telomeres and telomerase in cancer. Nat Rev Gastroenterol Hepatol 16: 544-558, 2019.

2. Kudo M: Systemic therapy for hepatocellular carcinoma: Latest advances. Cancers (Basel) 10: 412, 2018.

3. Ricke J, Klumpen HJ, Amthauer H, Bargellini I, Bartenstein P, de Toni EN, Gasbarrini A, Pech M, Peck-Radosavljevic M, Popovič $\mathrm{P}$, et al: Impact of combined selective internal radiation therapy and sorafenib on survival in advanced hepatocellular carcinoma. J Hepatol 71: 1164-1174, 2019.

4. Moehler M, Heo J, Lee HC, Tak WY, Chao Y, Paik SW, Yim HJ, Byun KS, Baron A, Ungerechts G, et al: Vaccinia-based oncolytic immunotherapy Pexastimogene Devacirepvec in patients with advanced hepatocellular carcinoma after sorafenib failure: A randomized multicenter Phase IIb trial (TRAVERSE). Oncoimmunology 8: 1615817, 2019.

5. Topalian SL, Hodi FS, Brahmer JR, Gettinger SN, Smith DC, McDermott DF, Powderly JD, Sosman JA, Atkins MB, Leming PD, et al: Five-year survival and correlates among patients with advanced melanoma, renal cell carcinoma, or Non-small cell lung cancer treated with nivolumab. JAMA Oncol 5: 1411-1420, 2019

6. Qazi MA, Vora P, Venugopal C, Sidhu SS, Moffat J, Swanton C and Singh SK: Intratumoral heterogeneity: Pathways to treatment resistance and relapse in human glioblastoma. Ann Oncol 28: 1448-1456, 2017. 
7. McDonald KA, Kawaguchi T, Qi Q, Peng X, Asaoka M, Young J, Opyrchal M, Yan L, Patnaik S, Otsuji E and Takabe K: Tumor heterogeneity correlates with less immune response and worse survival in breast cancer patients. Ann Surg Oncol 26: 2191-2199, 2019.

8. Wang Z, Chen J, Hu J, Zhang H, Xu F, He W, Wang X, Li M, Lu W, Zeng G, et al: cGAS/STING axis mediates a topoisomerase II inhibitor-induced tumor immunogenicity: J Clin Invest 129: 4850-4862, 2019.

9. Lindström LS, Yau C, Czene K, Thompson CK, Hoadley KA, Van't Veer LJ, Balassanian R, Bishop JW, Carpenter PM, Chen YY, et al: Intratumor heterogeneity of the estrogen receptor and the long-term risk of fatal Breast cancer. J Natl Cancer Inst 110: 726-733, 2018

10. Nassar D and Blanpain C: Cancer stem cells: Basic concepts and therapeutic implications. Annu Rev Pathol 11: 47-76, 2016.

11. Zhang J, Fujimoto J, Zhang J, Wedge DC, Song X, Zhang J, Seth S, Chow CW, Cao Y, Gumbs C, et al: Intratumor heterogeneity in localized lung adenocarcinomas delineated by multiregion sequencing. Science 346: 256-259, 2014.

12. Jackson CM, Choi J and Lim M: Mechanisms of immunotherapy resistance: Lessons from glioblastoma. Nat Immunol 20 : $1100-1109,2019$

13. Zhang Q, Lou Y, Yang J, Wang J, Feng J, Zhao Y, Wang L, Huang $\mathrm{X}, \mathrm{Fu} \mathrm{Q}, \mathrm{Ye} \mathrm{M}$, et al: Integrated multiomic analysis reveals comprehensive tumour heterogeneity and novel immunophenotypic classification in hepatocellular carcinomas. Gut 68: 2019-2031,2019.

14. Mroz EA and Rocco JW: MATH, a novel measure of intratumor genetic heterogeneity, is high in poor-outcome classes of head and neck squamous cell carcinoma. Oral Oncol 49: 211-215, 2013.

15. Rajput A, Bocklage T, Greenbaum A, Lee JH and Ness SA: Mutant-allele tumor heterogeneity scores correlate with risk of metastases in colon cancer. Clin Colorectal Cancer 16: e165-e170, 2017.

16. Wang Y, Feng W and Liu P: Genomic pattern of intratumor heterogeneity predicts the risk of progression in early stage diffuse large B-cell lymphoma. Carcinogenesis 40: 1427-1434, 2019.

17. Yarchoan M, Xing D, Luan L, Xu H, Sharma RB, Popovic A, Pawlik TM, Kim AK, Zhu Q, Jaffee EM, et al: Characterization of the immune microenvironment in hepatocellular carcinoma Clin Cancer Res 23: 7333-7339, 2017.

18. Nagarsheth N, Wicha MS and Zou W: Chemokines in the cancer microenvironment and their relevance in cancer immunotherapy. Nat Rev Immunol 17: 559-572, 2017.

19. Atretkhany KSN, Drutskaya MS, Nedospasov SA, Grivennikov SI and Kuprash DV: Chemokines, cytokines and exosomes help tumors to shape inflammatory microenvironment. Pharmacol Ther 168: 98-112, 2016

20. Alifano M, Mansuet-Lupo A, Lococo F, Roche N, Bobbio A, Canny E, Schussler O, Dermine H, Régnard JF, Burroni B, et al: Systemic inflammation, nutritional status and tumor immune microenvironment determine outcome of resected non-small cell lung cancer. PLoS One 9: e106914, 2014.

21. Wang L, Zhu B, Zhang M and Wang X: Roles of immune microenvironment heterogeneity in therapy-associated biomarkers in lung cancer. Semin Cell Dev Biol 64: 90-97, 2017.

22. Newman AM, Liu CL, Green MR, Gentles AJ, Feng W, Xu Y, Hoang CD, Diehn M and Alizadeh AA: Robust enumeration of cell subsets from tissue expression profiles. Nat Methods 12: 453-457, 2015.

23. Ye L, Zhang T, Kang Z, Guo G, Sun Y, Lin K, Huang Q, Shi X, Ni Z, Ding N, et al: Tumor-infiltrating immune cells act as a marker for prognosis in colorectal cancer. Front Immunol 10 2368,2019

24. Chen H, Chong W, Teng C, Yao Y, Wang X and Li X: The immune response-related mutational signatures and driver genes in non-small-cell lung cancer. Cancer Sci 110: 2348-2356, 2019.

25. Subramanian A, Tamayo P, Mootha VK, Mukherjee S, Ebert BL, Gillette MA, Paulovich A,Pomeroy SL, Golub TR, Lander ES and Mesirov JP: Gene set enrichment analysis: A knowledge-based approach for interpret-ing genome-wide expression profiles. Proc Natl Acad Sci USA 102: 15545-15550, 2005.

26. Shannon P, Markiel A, Ozier O, Baliga NS, Wang JT, Ramage D, Amin N, Schwikowski B and Ideker T: Cytoscape: A software environment for integrated models of biomolecular interaction networks. Genome Res 13: 2498-2504, 2003.
27. Langfelder $\mathrm{P}$ and Horvath S: WGCNA: An R package for weighted correlation. network analysis. BMC Bioinformatics 9: $559,2008$.

28. Hu K and Chen F: Identification of significant pathways in gastric cancer based on protein-protein interaction networks and cluster analysis. Genet Mol Biol 35: 701-708, 2012.

29. Komohara Y, Fujiwara Y, Ohnishi K and Takeya M Tumor-associated macrophages: Potential therapeutic targets for anti-cancer therapy. Adv Drug Deliv Rev 99: 180-185, 2016.

30. Prieto J, Melero I and Sangro B: Immunological landscape and immunotherapy of hepatocellular carcinoma. Nat Rey Gastroenterol Hepatol 12: 681-700, 2015.

31. McGranahan N, Furness AJ, Rosenthal R, Ramskov S, Lyngaa R, Saini SK, Jamal-Hanjani M, Wilson GA, Birkbak NJ, Hiley CT, et al: Clonal neoantigens elicit $\mathrm{T}$ cell immunoreactivity and sensitivity to immune checkpoint blockade. Science 351 : 1463-1469, 2016

32. Mannino MH, Zhu Z, Xiao H, Bai Q, Wakefield MR and Fang Y: The paradoxical role of IL-10 in immunity and cancer. Cancer Lett 367: 103-107, 2015.

33. Lippitz BE: Cytokine patterns in patients with cancer: A systematic review. Lancet Oncol 14: e218-e228, 2013.

34. Dinarello CA: Overview of the IL-1 family in innate inflammation and acquired immunity. Immunol Rev 281: 8-27, 2018.

35. Apte RN, Dotan S, Elkabets M, White MR, Reich E, Carmi Y, Song X, Dvozkin T, Krelin Y and Voronov E: The involvement of IL-1 in tumorigenesis, tumor invasiveness, metastasis and tumor-host interactions. Cancer Metastasis Rev 25: 387-408, 2006.

36. Li Y, Jia S and Dai W: Fisetin modulates human oral squamous cell carcinoma proliferation by Blocking $\mathrm{PAK}_{4}$ signaling pathways. Drug Des Devel Ther 14: 773-782, 2020.

37. Costa TDF, Zhuang T, Lorent J, Turco E, Olofsson $\mathrm{H}$, Masia-Balague M, Zhao M, Rabieifar P, Robertson N, Kuiper R, et al: PAK4 suppresses RELB to prevent senescence-like growth arrest in breast cancer. Nat Commun 10: 3589 , 2019.

38. Xu HT, Lai WL, Liu HF, Wong LLY, Ng IOL and Ching YP: PAK4 phosphorylates p53 at serine 215 to promote liver cancer metastasis. Cancer Res 76: 5732-5742, 2016.

39. Li X, Ke Q, Li Y, Liu F, Zhu G and Li F: DGCR6L, a novel PAK4 interaction protein, regulates PAK4-mediated migration of human gastric cancer cell via LIMK1. Int J Biochem Cell Biol 42: 70-79, 2010.

40. Rohr-Udilova N, Klinglmuller F, Schulte-Hermann R, Stift J, Herac M, Salzmann M, Finotello F, Timelthaler G, Oberhuber G, Pinter M, et al: Deviations of the immune cell landscape between healthy liver and hepatocellular carcinoma. Sci Rep 8: 6220, 2018.

41. Foerster F, Hess M, Gerhold-Ay A, Marquardt JU, Becker D, Galle PR, Schuppan D, Binder H and Bockamp E: The immune contexture of hepatocellular carcinoma predicts clinical outcome. Sci Rep 8: 5351, 2018.

42. Zong Z, Zou J, Mao R, Ma C, Li N, Wang J, Wang X, Zhou H, Zhang L and Shi Y: M1 Macrophages induce PD-L1 expression in hepatocellular carcinoma cells through IL-1 $\beta$ signaling. Front Immunol 10: 1643, 2019.

43. Lin EY, Li JF, Gnatovskiy L, Deng Y, Zhu L, Grzesik DA, Qian H, Xue XN and Pollard JW: Macrophages regulate the angiogenic switch in a mouse model of breast cancer. Cancer Res 66: 11238-11246, 2006.

44. Qian B, Deng Y, Im JH, Muschel RJ, Zou Y, Li J, Lang RA and Pollard JW: A distinct macrophage population mediates metastatic breast cancer cell extravasation, establishment and growth. PLoS One 4: e6562, 2009.

45. Wanderley CW, Colón DF, Luiz JP, Oliveira FF, Viacava PR, Leite CA, Pereira JA, Silva CM, Silva CR, Silva RL, et al: Paclitaxel reduces tumor growth by reprogramming tumor-associated macrophages to an M1 profile in a TLR4-dependent manner. Cancer Res 78: 5891-5900, 2018.

46. Helm O, Held-Feindt J, Grage-Griebenow E, Reiling N, Ungefroren $\mathrm{H}$, Vogel I, Krüger U, Becker T, Ebsen M, Röcken C, et al: Tumor-associated macrophages exhibit pro- and anti-inflammatory properties by which they impact on pancreatic tumorigenesis. Int J Cancer 135: 843-861, 2014.

47. Wang H, Wang X, Li X, Fan Y, Li G, Guo C, Zhu F, Zhang L and Shi Y: CD68+HLA-DR+ M1-like macrophages promote motility of

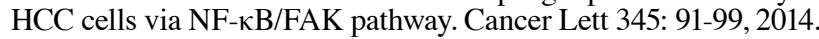

48. Wang N, Liang $\mathrm{H}$ and Zen K: Molecular mechanisms that influence the macrophage M1-M2 polarization balance. Front Immunol 5: 614, 2014 
49. Yuan A, Hsiao YJ, Chen HY, Chen HW, Ho CC, Chen YY, Liu YC, Hong TH, Yu SL, Chen JJ and Yang PC: Opposite effects of M1 and M2 macrophage subtypes on lung cancer progression. Sci Rep 5: 14273, 2015

50. Bardi GT, Smith MA and Hood JL: Melanoma exosomes promote mixed M1 and M2 macrophage polarization. Cytokine 105 63-72, 2018.

51. Imai K, Matsuyama S, Miyake S, Suga K and Nakachi K: Natural cytotoxic activity of peripheral-blood lymphocytes and cancer incidence: An 11-year follow-up study of a general population. Lancet 356: 1795-1799, 2000.

52. Quinn SR and O'Neill LA: The role of microRNAs in the control and mechanism of action of IL-10. Curr Top Microbiol Immunol 380: 145-155, 2014

53. Tian F, Yu C, Wu M, Wu X, Wan L and Zhu X: MicroRNA-191 promotes hepatocellular carcinoma cell proliferation by has circ_0000204/miR-191/KLF6 axis. Cell Prolif 52: e12635, 2019.

54. Kong XX, Lv YR, Shao LP, Nong XY, Zhang GL, Zhang Y, Fan HX, Liu M, Li X and Tang H: HBx-induced MiR-1269b in $\mathrm{NF}-\mathrm{kB}$ dependent manner upregulates cell division cycle 40 homolog (CDC40) to promote proliferation and migration in hepatoma cells. J Transl Med 14: 189, 2016.

55. Berthenet K, Boudesco C, Collura A, Svrcek M, Richaud S, Hammann A, Causse S, Yousfi N, Wanherdrick K, Duplomb L, et al: Extracellular HSP110 skews macrophage polarization in colorectal cancer. Oncoimmunology 5: e1170264, 2016.

56. Dorard C, de Thonel A, Collura A, Marisa L, Svrcek M, Lagrange A, Jego G, Wanherdrick K, Joly AL, Buhard O, et al: Expression of a mutant HSP110 sensitizes colorectal cancer cells to chemotherapy and improves disease prognosis. Nat Med 17: $1283-1289,2011$.
57. Wang XY, Li Y, Manjili MH, Repasky EA, Pardoll DM and Subjeck JR: Hsp110 over-expression increases the immunogenicity of the murine CT26 colon tumor. Cancer Immunol Immunother 51: 311-319, 2002.

58. Lu WT, Lemonidis K, Drayton RM and Nouspikel T: The Fanconi anemia pathway is downregulated upon macrophage differentiation through two distinct mechanisms. Cell Cycle 10: $3300-3310,2011$

59. Li J, Rao H, Jin C and Liu J: Involvement of the Toll-like receptor/Nitric oxide signaling pathway in the pathogenesis of cervical cancer caused by high-risk human papillomavirus infection. Biomed Res Int 2017: 7830262, 2017.

60. Hu HL, Shiflett LA, Kobayashi M, Chao MV, Wilson AC, Mohr I and Huang TT: TOP2 $\beta$-dependent nuclear DNA damage shapes extracellular growth factor responses via dynamic AKT phosphorylation to control virus latency. Mol Cell 74: 466-480.e4, 2019.

61. Romick-Rosendale LE, Hoskins EE, Privette Vinnedge LM, Foglesong GD, Brusadelli MG, Potter SS, Komurov K, Brugmann SA, Lambert PF, Kimple RJ, et al: Defects in the fanconi anemia pathway in head and neck cancer cells stimulate tumor cell invasion through DNA-PK and Rac1 signaling. Clin Cancer Res 22: 2062-2073, 2016.

This work is licensed under a Creative Commons Attribution-NonCommercial-NoDerivatives 4.0 International (CC BY-NC-ND 4.0) License. 